\title{
Obstacle course: Users’ maneuverability and movement efficiency when using Otto Bock C-Leg, Otto Bock 3R60, and CaTech SNS prosthetic knee joints
}

\author{
Margrit R. Meier, PhD; ${ }^{1-2^{*}}$ Andrew H. Hansen, PhD; ${ }^{2-3}$ Steven A. Gard, PhD; ${ }^{2,4-5}$ Angus K. McFadyen, PhD $^{6}$ \\ ${ }^{1}$ Department of Occupational Therapy and Prosthetics and Orthotics, Faculty of Health Sciences, Oslo and Akershus \\ University College of Applied Sciences, Oslo, Norway; ${ }^{2}$ Department of Physical Medicine and Rehabilitation, North- \\ western University Prosthetics-Orthotics Center, Feinberg School of Medicine, Northwestern University, Chicago, IL; \\ ${ }^{3}$ Minneapolis Department of Veterans Affairs (VA) Health Care System, Minneapolis, MN; ${ }^{4}$ Department of Biomedical \\ Engineering, McCormick School of Engineering and Applied Science, Northwestern University, Chicago, IL; ${ }^{5}$ Jesse \\ Brown VA Medical Center, Chicago, IL; ${ }^{6}$ School of Engineering and Computing, Glasgow Caledonian University, \\ Glasgow, Scotland, UK
}

\begin{abstract}
The performance and movement efficiency of prosthesis users while traversing a multisectional obstacle course (OC) were evaluated using a crossover design with random allocation of three prosthetic knee joints: the SNS (CaTech; Dayton, Ohio) the C-Leg (Otto Bock; Duderstadt, Germany), and the 3R60 (Otto Bock). Twelve users completed the OC twice with each joint, once without and once with a mental loading task (MLT). The performance was objectively assessed using time measurement from digital video recordings, and the Total Heart Beat Index was used to estimate movement efficiency. A 1 mo familiarization period was provided for each knee joint before data collection. It took longer to complete the OC with the 3R60 compared with either the SNS or the C-Leg. No significant time differences were found between the C-Leg and the SNS, but differences between the 3R60 and the SNS (slalom and rock sections) and between the 3R60 and the C-Leg (rock section) were observed. Within the simulated sand section, two participants fell with the C-Leg, one with the 3R60, and none with the SNS. Movement efficiency without MLT was similar between all joints, but with an MLT a significant decrease in movement efficiency was observed with the C-Leg. Previous experience using an SNS had no influence on the results.
\end{abstract}

Key words: 3R60, C-Leg, maneuverability, mental loading task, movement efficiency, obstacle course, prosthetic knee joint, SNS, Total Heart Beat Index, walking.

\section{INTRODUCTION}

More and more research is now available that demonstrates the effect of microprocessor-controlled prosthetic knee joints on user performance, satisfaction, and experiences. A recent review of the C-Leg (Otto Bock; Duderstadt, Germany) summarized these results [1]. Generally, the majority of available research presents results of studies with a focus on level and/or indoor walking. There are only a few studies available that present in-depth analysis of a particular activity over uneven surfaces, such as obstacle

\footnotetext{
Abbreviations: ANOVA $=$ analysis of variance, ECG = electrocardiography, $\mathrm{MLT}=$ mental loading task, $\mathrm{OC}=$ obstacle course, $\mathrm{PCI}=$ Physiological Cost Index, $\mathrm{pSNS}=$ previous SNS experience, SWOC $=$ standardized walking OC, THBI $=$ Total Heart Beat Index, VA = Department of Veterans Affairs, VACMARL = VA Chicago Motion Analysis Research Laboratory. *Address all correspondence to Margrit R. Meier, PhD; Oslo and Akershus University College of Applied Sciences, Faculty of Health Sciences, Department of Occupational Therapy and Prosthetics and Orthotics, Postboks 4, St Olavs plass, 0130 Oslo, Norway.

Email: Margrit-R.Meier@hioa.no http://dx.doi.org/10.1682/JRRD.2010.05.0094
} 
course (OC) performance [2] or stair ascent/descent [3-5], yet many different surface types are encountered during a single day. Depending on where a person is living, he or she may encounter different indoor surfaces in his or her home, such as carpeted, wooden, or tiled flooring. In addition, indoor stairs made from different materials with different raiser heights and configurations need to be handled, such as single flight stairs, corner stairs with multiple flights, or spiral stairs. Once outside the home, the variety of surfaces increases: in addition to man-made surfaces, such as walkways, natural surfaces need to be traversed to participate fully in life activities. Walkways come in a variety of compositions, such as cobblestones, concrete slabs, or pebble stones of different sizes, to name a few. Walkways can be level, inclined, or side-wise elevated, and their characteristic may change frequently over relatively short distances. Thus, studying maneuverability on nonlevel surfaces is important to many daily activities of prosthesis users and can add significantly to the understanding of possible interactions between walking surfaces and the performance characteristics of different prosthetic components. Clinical experiences strongly suggest that prosthesis users are sensitive to surface features during walking and standing. Given microprocessor-controlled knee joints' range of possible settings and their adaptation to walking speed, uneven terrain may be a domain where they provide superior maneuverability than passive, mechanicallycontrolled prosthetic knee joints. In particular, we were interested in determining how one of the microprocessorcontrolled prosthetic knee joints available on the market, the C-Leg, performed when prosthesis users walked over uneven surfaces. Because different surfaces may have different effects on maneuverability, an OC was developed with a number of different independent sections. Such a setup allowed for an analysis of possible interactions between walking surface and maneuverability in each section.

We only found a single published study that carefully analyzed the performance of users of transfemoral prostheses while they traversed an OC with different walking surfaces. Seymour et al. analyzed the C-Leg's performance on a standardized OC [2]. The validated standardized walking OC (SWOC) originally developed by Taylor and Gunther [6] was used. At the start of the SWOC, the participant sat in a chair with arms, then stood up, walked several feet straight ahead, turned right, stepped over an axillary crutch, continued walking, turned left, and walked across a multicolored mat. A right-turn followed, after which the participant navigated around a large kitchen trash can, followed by a walk across a heavy shag rug. The SWOC was completed when the participant sat down on an armless chair placed at the end of the course. Study participants completed the SWOC under two conditions: (1) hands free and (2) with a weighted laundry basket (4.5 kg) using their previous nonmicroprocessor knee joints and their current C-Legs. Under the hands-free condition, the participants completed the SWOC significantly faster with the C-Leg than with the previous knee joint, taking significantly fewer steps and producing significantly fewer step-offs. No stumbles were recorded with either knee joint. Under the weighted laundry basket condition, no differences were observed between the knee joints except in one variable: total time taken to complete the SWOC was shorter with the C-Leg than with the nonmicroprocessor knee joints. Perhaps more differences between the knee joints would have been observed if the considerably different durations of prior experience with the C-Leg had been controlled for. Prior experience with the C-Leg varied between 2 and 44 months. Seymour et al. also measured oxygen consumption of subjects walking on a treadmill (CardioO2/EGG Exercise System, Medical Graphics Corporation; St. Paul, Minnesota), but not while participants ambulated on the SWOC [2].

The purpose of our study was to evaluate the maneuverability and movement efficiency of transfemoral prosthesis users traversing an OC with defined surface characteristics while wearing three different prosthetic knee joints: the C-Leg, the SNS unit (CaTech; Dayton, Ohio), and the 3R60 prosthetic knee joint (Otto Bock). Based on the documented favorable user perceptions of microprocessor-controlled prosthetic knee joints [7], we hypothesized that OC performance with the C-Leg would be best, i.e., that persons using the C-Leg would traverse the OC in the shortest time. We also hypothesized that movement efficiency with the C-Leg would be highest since it has been previously reported that microprocessor-controlled knee joints reduce metabolic energy expenditure in the lower and self-selected speed range [8-9].

\section{METHODS}

\section{Participants' Eligibility Criteria}

Persons with transfemoral amputation, between 40 and 60 years old, with a body mass less than $125 \mathrm{~kg}$ 
(because of the components' weight limitations) were included if they presented with no serious complications that interfered with their walking ability, had 6 or more months of experience with a definitive prosthesis, were able to walk unassisted (i.e., independently, no crutches or walking aids) at a comfortable speed without undue fatigue and without health risk, and were able to negotiate stairs. Individuals whose prostheses demonstrated a poor socket fit were excluded from the study.

\section{Fitting of Test Prosthesis and Study Design}

A test prosthesis was fabricated for each participant using a duplication of his or her current prosthetic socket and a Dynamic Plus foot (Otto Bock), a foot approved by the manufacturer to be used in combination with the CLeg. The three knee joints used for this study were (1) the Otto Bock C-Leg, a fluid-controlled single-axis prosthetic knee joint whose onboard microprocessor controls stance and swing phase; (2) the 3R60 prosthetic knee joint from Otto Bock, a multi-axis fluid-controlled prosthetic knee joint with a mechanical swing-phase control only; and (3) the Mauch SNS prosthetic knee joint from CaTech, a single-axis fluid-controlled knee joint whose swing- and stance-phase damping are mechanically controlled [10]. Participants wore each prosthetic knee joint for an accommodation period of 4 weeks before testing [11]. The study had a crossover design; after each testing session, the current prosthetic test knee joint was exchanged with the next test knee joint and aligned by an experienced prosthetist before the participant was sent home for the next accommodation period. Throughout the dynamic alignment session, participants received verbal instruction on how to use the current test joint appropriately. For the C-Leg, however, the participants received additional written instructions in the form of an information brochure from Otto Bock about the C-Leg. All participants received the same information. Dynamic alignment was carried out on a level walking surface using observational gait analysis, as is common in general clinical practice. Dynamic alignment and instructions were given by the same prosthetist and continued until both the participant and the prosthetist were confident that the knee could be used dynamically and without undue anxiety by the subject. For the duration of the accommodation period, study participants were encouraged to contact the prosthetist any time for additional fine-tuning. None of the study participants requested additional fine-tuning sessions. The sequence of fitting the three knee joints was randomized.

\section{Determination of Variables}

The two main outcomes of the study were (1) time as a measure of OC performance and (2) Total Heart Beat Index (THBI) [12] as a measure of movement efficiency. The time to complete the OC was used as a measure of performance for the different prosthetic knee joints. In addition, any falls were recorded. The THBI is a validated index of movement efficiency in children with cerebral palsy. This index was chosen for its strength of measuring movement efficiency without the need to reach a plateau heart beat as is required, for example, with the Physiological Cost Index (PCI). In our case, completing the OC required a relatively short time, a period that was insufficient for subjects to reach steady heart rate and thus excluded the use of the PCI. The THBI is calculated by dividing the total number of heart beats measured over a specified time period by the total distance traveled during that time. As THBI increases, movement efficiency decreases.

\section{Experimental Protocol}

Study participants were asked to complete the OC twice: first without a mental loading task (MLT) and then with an MLT. For safety reasons, all participants started without the MLT. The OC was set up in the Department of Veterans Affairs (VA) Chicago Motion Analysis Research Laboratory (VACMARL). It consisted of seven sections: (1) a foam section (3 $\mathrm{m}$ long, $1 \mathrm{~m}$ wide, and $15 \mathrm{~cm}$ high with a 20 Durometer on a shore A scale), (2) a narrow slalom section around three chairs each measuring $0.58 \mathrm{~m}$ across and spaced equally at a distance of roughly $0.5 \mathrm{~m}$, (3) a vacuumized beanbag section simulating sand (3 m long, $1 \mathrm{~m}$ wide), (4) a rock section ( $3 \mathrm{~m}$ long, $1 \mathrm{~m}$ wide; field rocks of different sizes and shapes, average rock size roughly fist-size), (5) a short sloping ramp (1.5 m long, $1.4 \mathrm{~m}$ wide, $5^{\circ}$ downward angle), (6) a $90^{\circ}$ left turn, and (7) a final stair step (height: $12 \mathrm{~cm}$ ). A handrail on the left-hand side was present for both the ramp and the step section. The OC measured a total length of $23.2 \mathrm{~m}$ from start to finish (Figure 1).

The MLT consisted of an arithmetic calculation in which the participant was required to count vocally backward in 3-step decrements using the first knee joint during the first visit, in 7-step decrements using the second knee joint during the second visit, and in 3-step decrements using the third knee joint during the third visit. As previously mentioned, the knee joints were randomly assigned. The three-digit MLT starting number was specifically selected 


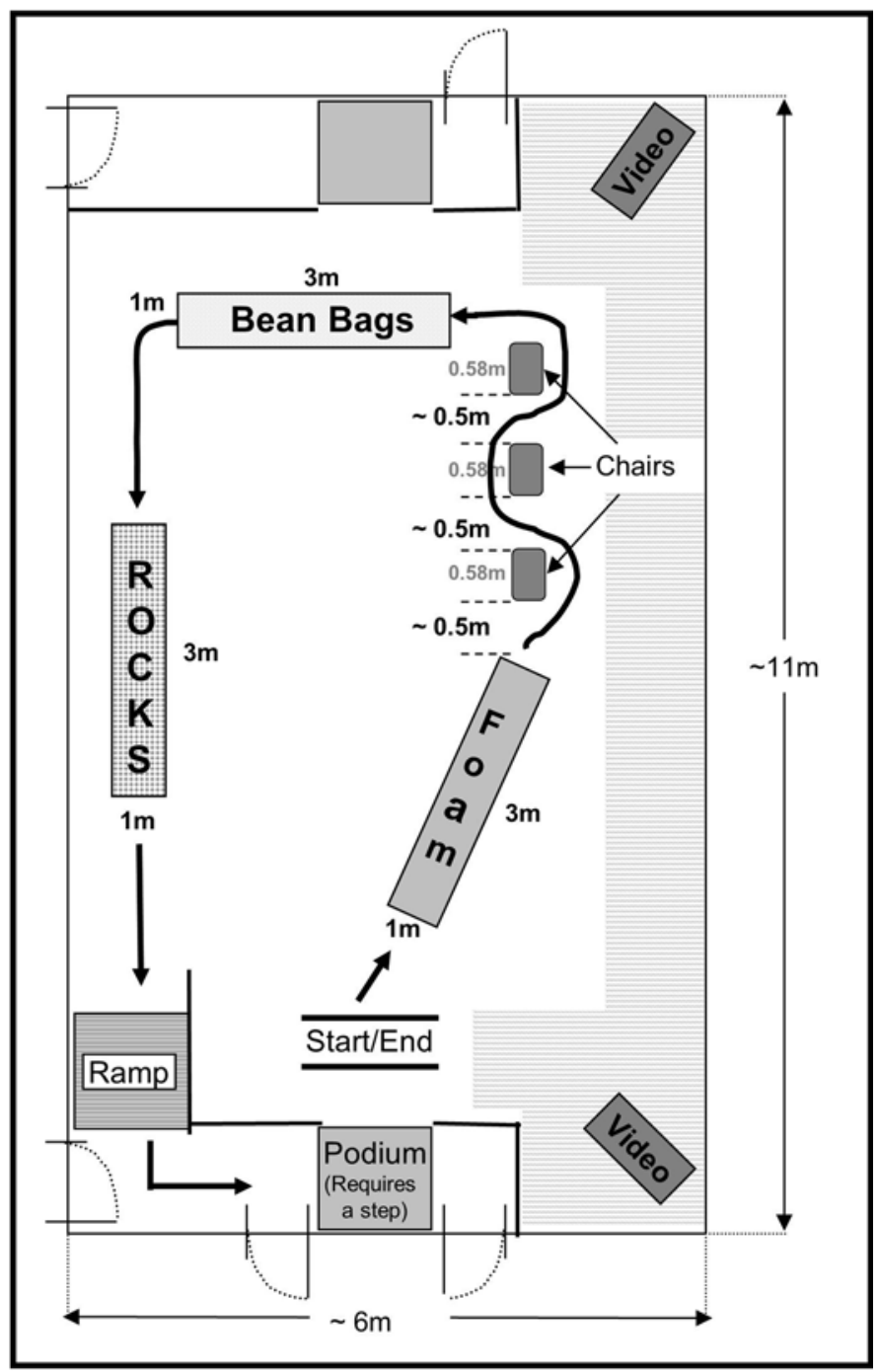

Figure 1.

Overview of obstacle course (OC) setup within laboratory. Foam section ( $1 \mathrm{~m}$ wide, $3 \mathrm{~m}$ long, $15 \mathrm{~cm}$ high), slalom section around three chairs, vacuumized beanbags to mimic sand ( $1 \mathrm{~m}$ wide, $3 \mathrm{~m}$ long), rock section ( $1 \mathrm{~m}$ wide, $3 \mathrm{~m}$ long), ramp (1.4 m wide, $1.5 \mathrm{~m}$ long, $5^{\circ}$ downward slope), corner ( $90^{\circ}$ left turn) and step (12 cm high). Two video cameras were set up in such a way that entire OC could be filmed, allowing time measurements for each section.

to minimize repetition and to prevent participants reaching zero or negative numbers before completion of the trial. The MLT for visits 1 and 3 had the same starting number; the MLT for visit 2 started with a higher number because of the larger arithmetic step decrement required during this visit. All participants received the same starting numbers.
No specific information was given to the participants other than to perform the MLT while traversing the OC. Time was objectively determined from a digitized videotape recording (Sony Super Steadyshot miniDV Handycam Vision; Tokyo, Japan) of the participants navigating the OC, allowing for time assessment of the different sections as well as the entire OC (resolution of $0.033 \mathrm{~s}$ between video frames). No familiarization run was allowed and no encouragement was given, nor were the participants informed that their traverse time was being measured. The instructions given were simply to complete the OC. To demonstrate the navigation path, the lead investigator walked the OC once as the participant watched. However, she did not walk over the beanbag section in order to avoid any visual feedback to the participants prior to their own experience. During the MLT run, the numbers spoken by the participants were recorded manually. Participants were aware that their answers were noted.

Each participant's heart rate was monitored using a Polar S610 heart rate monitor (Lake Success, New York). For heart rate analysis, the data were transferred from the receiver unit (wrist watch) to a computer and processed to extract the relevant heart rate. The heart rate monitor did not allow remote adding of tags into the data stream to delineate particular OC segments. Hence, the heart rate over the entire OC was analyzed instead of over the individual OC sections.

\section{Statistical Analysis}

A two-factor repeated measures analysis of variance (ANOVA) was used with task (without MLT, with MLT) and joints (3R60, SNS, C-Leg) as fixed factors and subjects as a random factor. The diagnostics for this parametric ANOVA model were satisfactory for all parameters. The dependent "time taken" variables during the beanbag and rock sections of the OC were determined to have skewed distributions; hence, a logarithmic (base 10) transformation was carried out and one (same subject) of the three outliers was removed in order to satisfy the parametric model. An outlier was regarded as a data point that lay beyond three standard deviations of the mean. The variable "total time" to complete the OC satisfied the model without transformation once the outliers were disregarded. Where necessary, post hoc analyses were performed using Bonferroni corrections to account for multiple comparisons. Because 50 percent of the participants had used an SNS unit prior to study entry, we decided to test if previous experience with the SNS unit had an influence on the 
performance. Data were regrouped into two groups: previous SNS experience (pSNS) and non-pSNS users (persons who did not have previous experience with the SNS). To test for any significance of this new variable, a third factor was added to the previous model and the analysis was repeated, this time as a three-factor repeated measures ANOVA. This reanalysis reduced the significant level to 0.02. Paired comparisons between no-MLT and with-MLT within each joint type were assessed using Wilcoxon signed rank tests given the skewed distributions of the variables. All tests were completed using the statistical software SPSS 15.0 (Chicago, Illinois).

\section{RESULTS}

A total of 24 participants were enrolled in the study. However, eight did not come in for either their initial appointment or the first testing session, two participants dropped out, and two asked to be withdrawn from the study. The final sample size of 12 participants was composed of 2 women and 10 men. All participants were experienced prosthesis users whose amputations occurred on average 21 years previously ( $\pm 15.8 \mathrm{yr}$ ), primarily as a result of a traumatic incident. Participants' characteristics are summarized in Table 1. Participants used a variety of

Table 1.

Participants' sociodemographic data $(n=12)$.

\begin{tabular}{lc}
\hline \multicolumn{1}{c}{ Category } & Value \\
\hline Sex $(n)$ & 2 \\
Female & 10 \\
Male & \\
Ethnic Background $(n)$ & 3 \\
$\quad$ African American & 9 \\
Caucasian & $46 \pm 8.6$ \\
Age, yr (Mean \pm SD) & $1.76 \pm 0.08$ \\
Height, m (Mean \pm SD) & $82.0 \pm 13.5$ \\
Weight, kg (Mean \pm SD) & 15.6 \\
Years Since Amputation & \\
Reason for Amputation ( $n$ ) & 2 \\
Congenital & 2 \\
Infection & 1 \\
PVD & 7 \\
Traumatic & 4 \\
$\quad$ Car Accident (inside vehicle) & 1 \\
Car Accident (outside vehicle) & 1 \\
Gunshot & 1 \\
Work Accident & \\
\hline PVD = peripheral vascular disease, SD = standard deviation. & \\
\hline \hline
\end{tabular}

different prosthetic components, with the CaTech SNS the most commonly used prosthetic knee joint in this study sample (Table 2).

Analyses of the total time taken to complete the OC revealed that joint $(p<0.001)$ and task $(p=0.04)$ had a significant influence on the total time participants spent completing the OC (Figure 2). However, the interaction between joint and task was not significant $(p=0.71)$, indicating that both were independent factors.

A detailed analysis of each OC section indicated that within the foam section only joint $(p=0.01)$, not task ( $p=$ 0.32 ), had a significant influence on the time required for participants to complete the section. There was a significant difference between the 3R60 and the C-Leg ( $p=$ 0.03 ), with the participants walking slightly slower with the 3R60 (6.16 $\pm 2.18 \mathrm{~s})$ than the C-Leg $(5.67 \pm 2.19 \mathrm{~s})$. However, no significant differences were found between the 3R60 (6.16 $\pm 2.18 \mathrm{~s})$ and the SNS $(5.82 \pm 2.22 \mathrm{~s})(p=$ $0.32)$ or between the C-Leg (5.67 $\pm 2.19 \mathrm{~s})$ and the SNS $(5.82 \pm 2.22 \mathrm{~s})(p=0.97)$. Also, there was no statistically significant interaction between joint and task $(p=0.07)$.

Task had no influence on the time needed to complete the slalom section $(p=0.12)$. There was also no interaction

Table 2.

Description of participants' prosthesis prior to study entry $(n=12)$.

\begin{tabular}{ll}
\hline \multicolumn{1}{c}{ Prosthesis } & $\boldsymbol{n}$ \\
\hline Prosthetic Feet & 1 \\
Ceterus & 1 \\
C-Walk & 1 \\
Endolite Multiflex & 1 \\
Flex-Reflex & 1 \\
Flex-Walk & 1 \\
Pathfinder & 2 \\
SACH & 1 \\
Seattle Light & 1 \\
Single-Axis (name unknown) & 1 \\
Total Concept & 1 \\
Other & 1 \\
\hline Prosthetic Knee & 1 \\
Otto Bock C-Leg & 6 \\
Otto Bock Constant Friction & 1 \\
SNS CaTech & 2 \\
Teh Lin Graph-Lite 5-Bar & 1 \\
Össur Total Knee & 9 \\
Other & 3 \\
\hline Prosthetic Socket & \\
IC-Type & \\
Quad-Type & \\
\hline IC = ischial containment, SACH = solid ankle cushion heel. & \\
\hline \hline
\end{tabular}




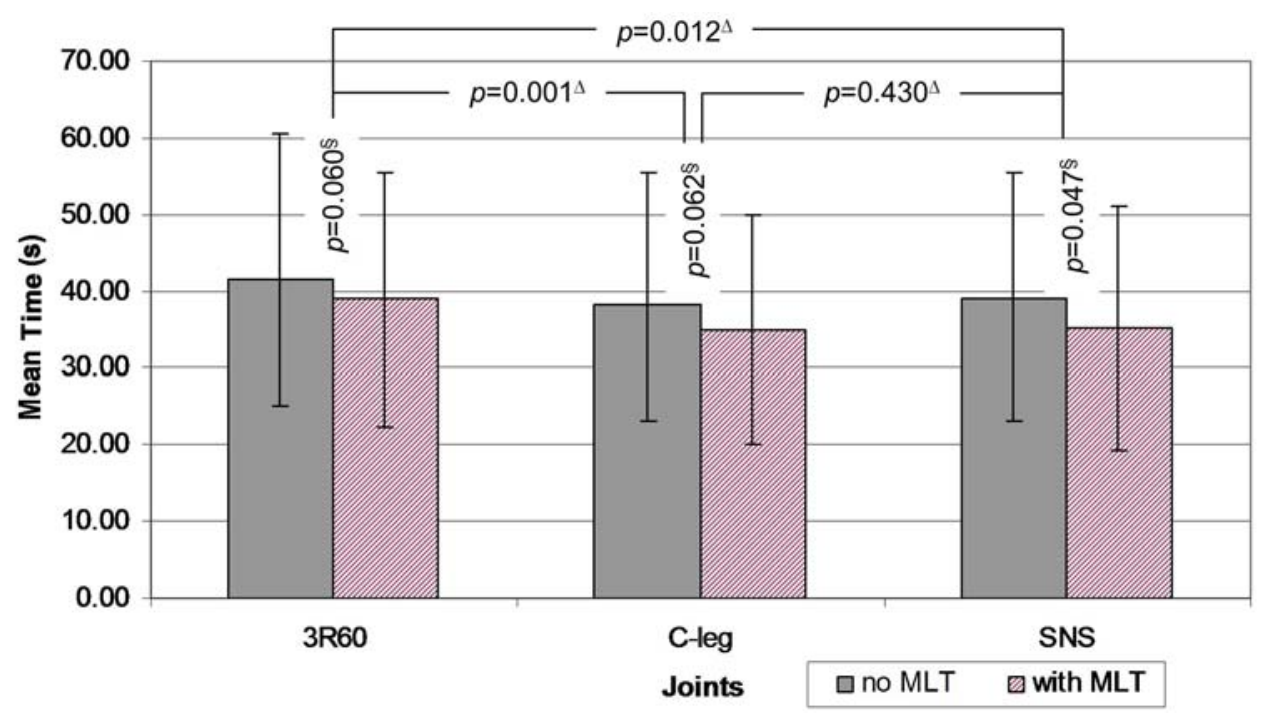

Figure 2.

Total time taken to complete obstacle course (OC). Time differences between tasks (with mental loading task [MLT] and no MLT) were significant only with SNS unit. Time differences exist only between 3R60 and C-Leg and between 3R60 and SNS. Participants maneuvering OC with C-Leg had similar time as when they were maneuvering OC with SNS. Actual and not transformed data are shown. ${ }^{\Delta} p$-Value associated with univariate analysis of variance. ${ }^{\S} p$-Value associated with Wilcoxon signed rank test.

between joint and task $(p=0.61)$. However, joint alone did have an influence on the time needed to complete the slalom section. There was a significant difference $(p=0.05)$ in the time between the 3R60 $(7.75 \pm 2.16 \mathrm{~s})$ and the SNS $(6.93 \pm 2.42 \mathrm{~s})$, with participants completing the slalom section slightly faster while wearing the SNS than while wearing the 3R60. No statistical differences were found between the 3R60 (7.75 $\pm 2.16 \mathrm{~s})$ and the C-Leg (6.99 \pm $2.61 \mathrm{~s})(p=0.06)$ or between the C-Leg $(6.99 \pm 2.61 \mathrm{~s})$ and the SNS (6.93 $\pm 2.42 \mathrm{~s})(p=0.99)$.

While maneuvering the beanbag section, three participants (25\%) fell when no MLT was applied. At the time, two of the fallers were using the C-Leg and the third was using the 3R60. No falls occurred when participants walked with the SNS unit. Participants who fell previously did not fall during the second round when the MLT was applied. Task had a significant influence on the time needed to complete the beanbag section $(p=0.001)$, with all participants walking significantly faster when the MLT was applied (Figure 3). However, joint had no statistical effect on completion time $(p=0.09)$. No interaction existed between joint and task ( $p=0.99)$.

Within the rock section, task $(p=0.45)$ and the interaction of task and joint $(p=0.39)$ had no statistically significant influence on completion time. However, joint was determined to have an influence with significant differences in completion times between the 3R60 and the SNS $(p=0.01)$ and between the 3R60 and the C-Leg $(p=$ 0.002 ). Participants required $7.24 \pm 3.65$ s to complete the rock section when fitted with the 3R60, but only required $5.64 \pm 2.69 \mathrm{~s}$ when fitted with the C-Leg. No statistical difference existed between the SNS $(6.15 \pm 3.73 \mathrm{~s})$ and the C-Leg (5.64 $\pm 2.69 \mathrm{~s})(p=0.99)$ (Figure 4).

No statistical differences were recorded between task ( $p=0.16)$, joint $(p=0.05)$, or their interaction $(p=0.84)$ for the ramp section. The same applies for the corner and step sections (Figure 5, Table 3).

Based on prior use of the SNS unit (50\% of the study participants had an SNS prior to study entry, Table 2), the variable pSNS was created by splitting the sample into two groups based on evaluation of participants' previous experiences. There were no statistically significant effects of pSNS on any of the OC sections or on total time. Also, all interactions of pSNS with task and pSNS with joint were statistically insignificant. Thus, previous experiences with an SNS unit did not appear to have had an influence on the measured outcomes (Table 4).

Task had an overall significant effect on the THBI ( $p=$ $0.02)$, but joint did not ( $p>0.99)$, suggesting that the type of knee joints participants were using when completing this 


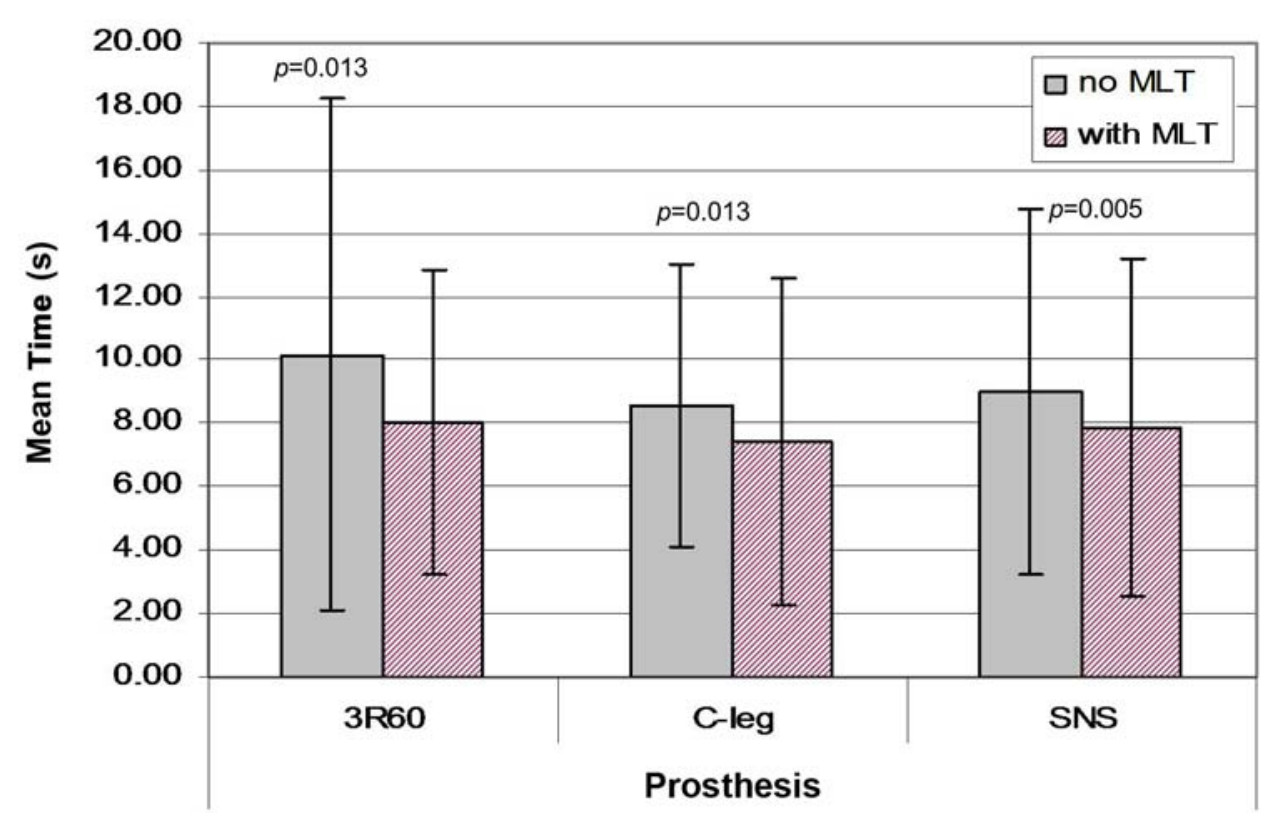

Figure 3.

Beanbag section-Influence of task on performance. All participants completed this section significantly faster when mental loading task (MLT) was applied. $p$-Values are associated with Wilcoxon signed rank test and Bonferroni correction to take into account multiple testing. Actual and not transformed data are shown.

OC did not have an influence on participants' movement efficiency. The interactions between task and joint $(p=$ $0.43)$, pSNS and task $(p=0.06)$, and pSNS and joint ( $p=$ 0.15 ) were all statistically insignificant, demonstrating that task alone seems to be responsible for the differences in THBI, irrespective of either knee joint type or previous experience with an SNS unit (Figure 6).

A Wilcoxon signed rank revealed that participants wearing the C-Leg demonstrated a significant increase in THBI $(p=0.047)$ when walking with the MLT (Figure 6), indicating a reduction in movement efficiency with the CLeg when subjects performed the MLT. A similar pattern was observed with the 3R60, but the THBI increase was not statistically significant $(p=0.16)$. The result for the SNS knee joint was unexpected: participants completing the OC with the MLT demonstrated a decrease in THBI, indicating an increase in movement efficiency. However, this difference was not statistically significant $(p=0.25)$.

\section{DISCUSSION}

Neither hypothesis-(1) OC performance would be best with the C-Leg, or (2) movement efficiency would be highest (lowest THBI) with the C-Leg-was supported by the results of our study. Statistically significant differences were observed only between the 3R60 and the CLeg and/or the 3R60 and the SNS, but not between the CLeg and the SNS. That is, participants completed the OC equally fast while walking with the SNS and the C-Leg.

A substantial ambulation challenge was observed within the beanbag section of the OC. Three falls occurred during the participants' first round of the OC (without MLT): two falls occurred in participants wearing the CLeg, one with the 3R60, and none with the SNS. The falls occurred in three subjects: two who were 54 years old and one who was 62 years old. All three had different etiologies of amputations ( 1 traumatic, 1 peripheral vascular disease, 1 infection), and had their amputations 3, 28, and 6 years prior to testing, respectively. We concluded that the falls were not due to the participants' characteristics, but were likely elicited by a combination of the particular knee joint they were using during that trial and the challenges imposed by the compliant surface they were traversing at that time. This finding is in contrast with other previously reported data. Hafner et al. obtained subjective feedback from prosthesis users who perceived a reduction in stumbles and falls while walking with the C-Leg compared with 


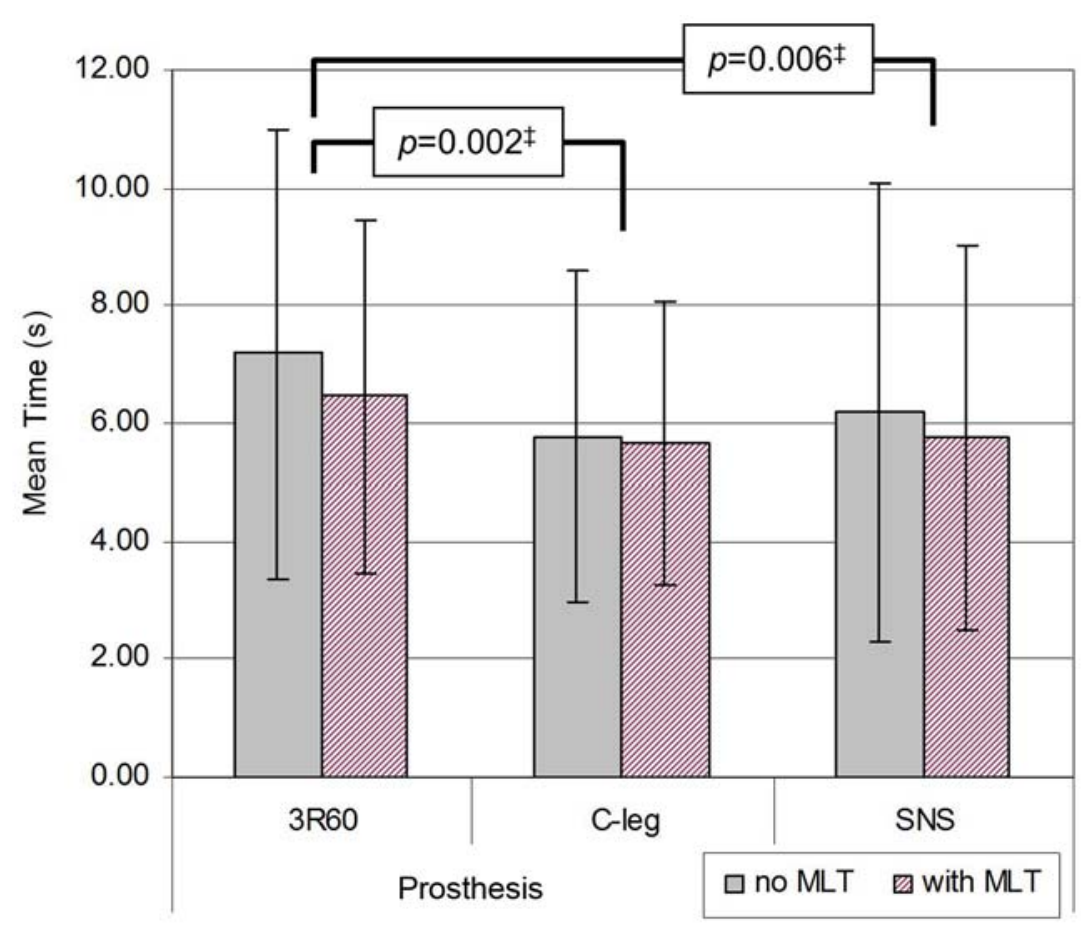

Figure 4.

Rock section-Influence of joint on performance. Only joint had significant influence on participants' performance during this section; significant differences were found between 3R60 and SNS and between 3R60 and C-Leg, but not between C-Leg and SNS. Actual and not transformed data are shown. ${ }^{\ddagger} p$-Value associated with univariate analysis of variance. MLT = mental loading task.

a mechanical knee [5]. However, our results indicate that there may be some terrain conditions that can make C-Leg users more prone to falls. The C-Leg requires a minimum forefoot loading of 66 percent body weight in late stance to trigger a reduction in knee flexion resistance, permitting knee break and initiating swing phase [13]. But this required loading appears to be difficult to achieve on surfaces that have higher compliance (i.e., lower stiffness), such as in the case of the beanbag section of the OC. When prosthesis users walk on relatively compliant surfaces, the C-Leg may remain extended in late stance phase when the knee joint should begin flexing in preparation for swing, leading to stumbles or falls. Therefore, the compliance is believed to have been the primary contributing factor to why the participants' falls occurred in the beanbag section. Frame-by-frame analysis of the video recording as those subjects traversed the beanbag section indicated that the initiation of the C-Leg's swing phase either did not occur or occurred at an inappropriate time, thus directly contributing to the falls. Although the manufacturer's specified require- ment for knee break to occur in late stance is at a minimum of 66 percent body weight loading on the "forefoot," the CLeg actually monitors an external dorsiflexion torque on the pylon. Even though the participants likely placed full body weight on their prosthesis when traversing the beanbag section, the compliant surface may not have permitted the center of pressure of the ground reaction force to sufficiently progress anterior under the prosthetic foot and create a sufficient lever arm to enable the ankle joint torque to reach the minimum threshold. In spite of our observations, it is possible that subjects in the Hafner et al. study actually experienced a lower incidence of stumbles and falls because they may not have encountered these types of compliant surfaces while using the C-Leg [5]. Therefore, we acknowledge that the C-Leg may improve stability in particular situations that would normally cause stumbles or falls with some mechanical knees. Nonetheless, our study provides additional information that is relevant for clinical use. If a patient routinely encounters compliant surfaces during his or her everyday use of his or her prosthesis, a 


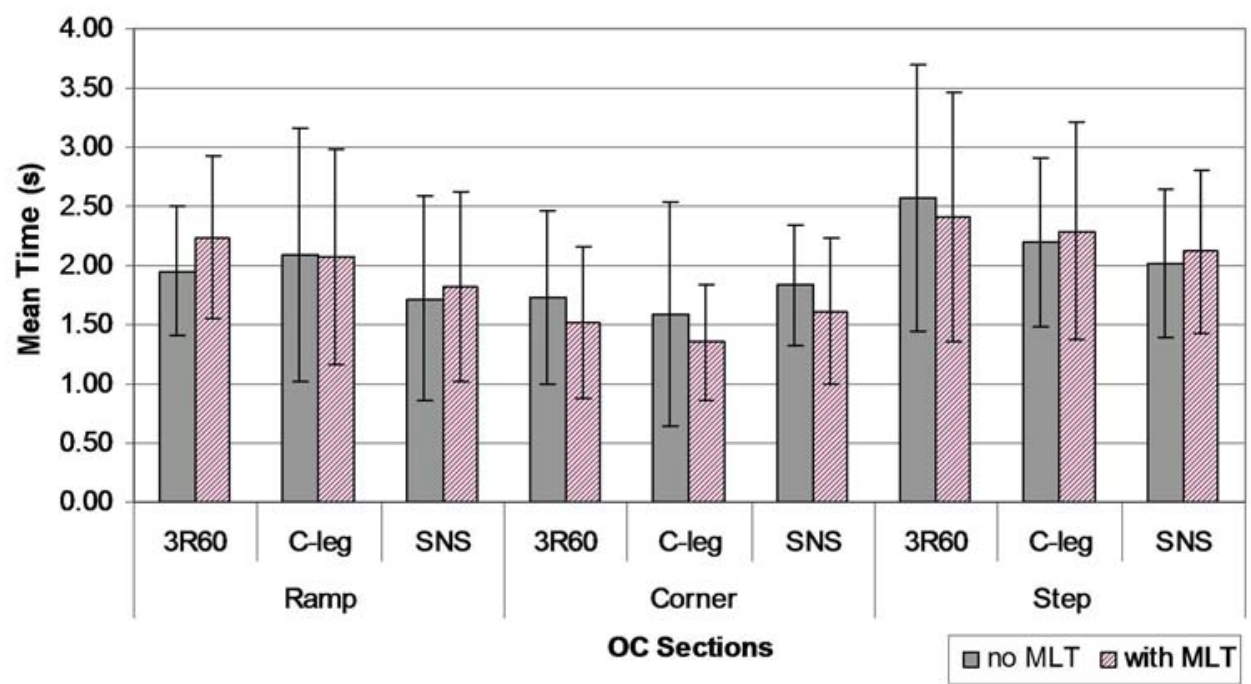

Figure 5.

Ramp, corner, and step sections_-Influence of task/joint on performance. Neither joint, task, nor interaction had significant influence on participants' performance during these three sections (see Table 3 for details). MLT = mental loading task, OC = obstacle course.

Table 3.

p-Values ${ }^{*}$ for last three sections of obstacle course.

\begin{tabular}{lccc}
\multicolumn{1}{c}{ Factor } & Ramp & Corner & Step \\
\hline Joint & 0.05 & 0.36 & 0.08 \\
Task & 0.16 & 0.09 & 0.95 \\
$\begin{array}{l}\text { Interaction Between } \\
\text { Joint and Task }\end{array}$ & 0.84 & 0.96 & 0.61 \\
\hline
\end{tabular}

${ }^{*}$ Associated with two-factor analysis of variance.

Joint (3-levels: 3R60, SNS, C-Leg), Task (2-levels: no mental loading task [MLT], with MLT).

C-Leg may not be the best choice for that individual, or else the prosthesis may need to be adjusted to reduce the risk of falls. The absence of falls during the MLT (the second trial across the OC) is most likely attributable to the learning effect by the subjects. The falls experienced by the users during their first trials on the OC may have made them particularly wary and cued them on how to react to these sections on subsequent trials to prevent additional falls.

It could be argued that the beanbag section is not representative of walking surfaces encountered in real life. However, the authors have heard anecdotal first-hand accounts from prosthesis users of stumbles occurring on soft terrains while walking with the C-Leg because of the inability of the knee to flex in late stance phase for swing. More research is required to determine the interaction of surface compliance and the function of microprocessorcontrolled knees in the late stance phase/early swing
Table 4.

Effect of previous SNS experience (pSNS) and interactions on OC.

\begin{tabular}{lccc}
\hline \multirow{2}{*}{ OC Section } & \multicolumn{3}{c}{$\boldsymbol{p}$-Value } \\
\cline { 2 - 4 } & pSNS & pSNS $\times$ Task & pSNS $\times$ Joint \\
\hline Foam & 0.41 & 0.72 & 0.96 \\
Slalom & 0.62 & 0.43 & 0.67 \\
Beanbag & 0.02 & 0.81 & 0.29 \\
Rock & 0.02 & 0.78 & 0.56 \\
Ramp & 0.56 & 0.05 & 0.22 \\
Corner & 0.20 & 0.35 & 0.77 \\
Step & 0.19 & 0.36 & 0.18 \\
Total Time & 0.30 & 0.71 & 0.57
\end{tabular}

${ }^{*}$ Associated with three-factor analysis of variance: pSNS (2-levels: Yes, No). Note: $\alpha=0.017$; adjustment of significant level was necessary due to re-analysis of data. See text for further details.

Joint (3-levels: 3R60, SNS, C-Leg), Task (2-levels: no mental loading task [MLT], with MLT).

OC $=$ obstacle course.

phase. Similarly, manufacturers are encouraged to carefully review and evaluate the control algorithms programmed into their microprocessor-controlled knees and consider the effect of surface stiffness and uneven terrains on knee function and performance.

The time differences in the rock section were attributable only to the different knee joints. When participants were walking with the C-Leg, they were able to complete this section fastest, although there was not a significant difference in times between the C-Leg and the SNS. It could 


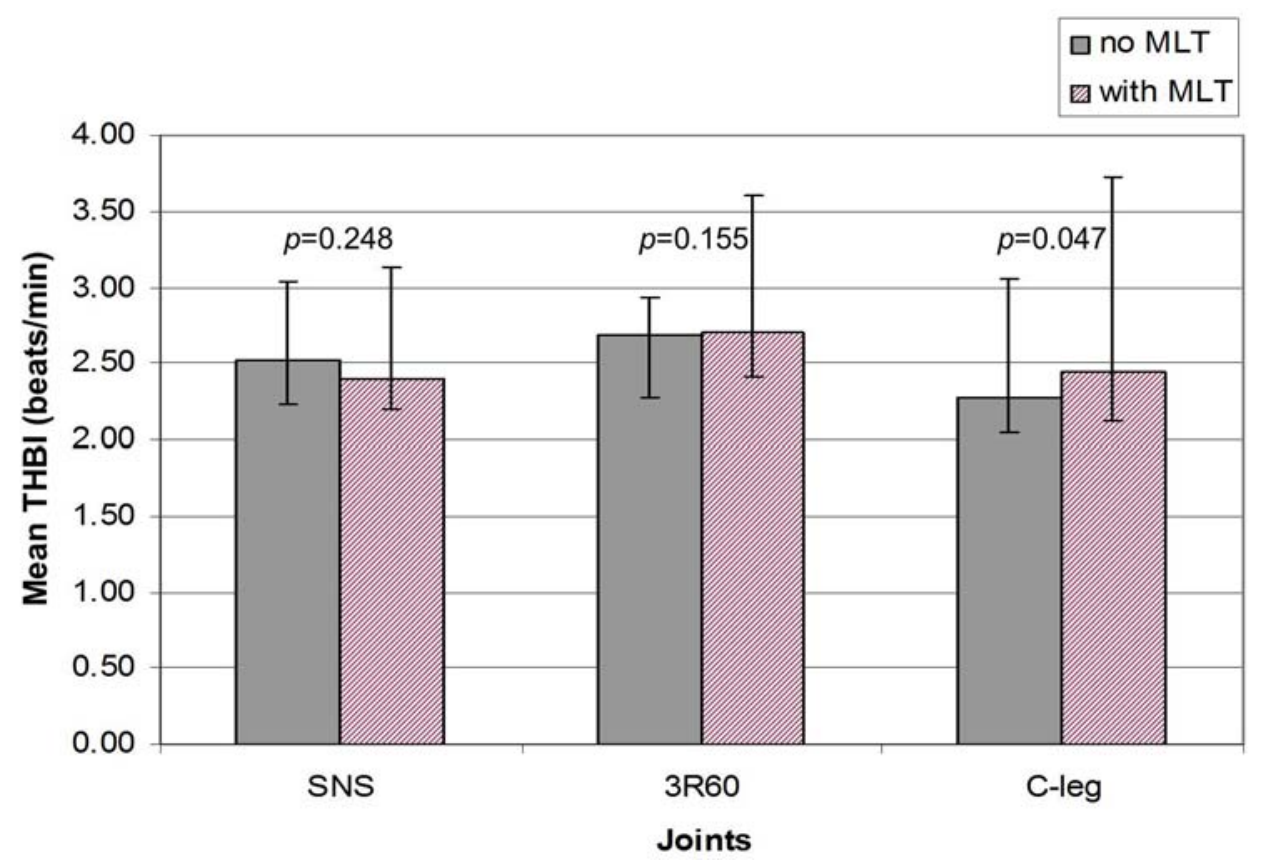

Figure 6.

Total Heart Beat Index (THBI), estimate for movement efficiency. Unexpected slight, but statistically significant, increase in THBI (i.e., decrease of movement efficiency) between tasks was detected with C-Leg. No significant overall differences were observed between joints. $p$-Value associated with Wilcoxon signed rank test. MLT = mental loading task

be argued that the section surfaces directly contributed to this result. Effects of different surfaces on gait have been analyzed in young and elderly populations [14-19]. Irregular surfaces and obstacle-clearing tasks increase the variability of gait parameters, widening step width and length in all age groups and necessitating compensatory movements. In addition, as the task becomes more complex, the time required to complete the task increases independent of age group. Few publications report the effect of different surface types on the gait of people wearing prostheses $[5,20]$. In general, previous work has concentrated on determining the effect of different prosthetic knee joints on walking performance, but they have generally provided no clear indication of how a particular knee joint affects maneuverability [5]. Yang et al. demonstrated that persons with transfemoral amputations are at higher risk of falling irrespective of the type of surface they encounter and independent of whether a slip occurs on the prosthetic or nonamputated side [20]. A cursory comparison of results from the current study with one that investigated nondisabled elderly subjects walking over a similar obstacle course [19] revealed that the transfemoral prosthesis users required approximately twice as much time to walk over uneven terrain than the nondisabled elderly despite being 28 years younger on average. Perhaps a different approach in gait training focusing on how uneven terrain can successfully be managed - emotionally, psychologically, and physically, irrespective of the fitted knee joint—would produce the desired outcome, which is a successful navigation irrespective of a person's age [21].

Within the ramp, corner, and step sections, participants maneuvered similarly regardless of the knee joint used. These results suggest that neither task nor joint type influenced the time required to traverse these sections, as no significant differences were observed. However, the different sections may have been too close together and/ or too short in length to discriminate discernible differences within the variables analyzed.

Hafner et al. compared the C-Leg with nonmicroprocessor-controlled knee joints and reported nonsignificant results when participants walked on uneven ground at their self-selected walking speed [5]. Their results suggested that the C-Leg may be beneficial for participants while negotiating uneven terrain since their walking time to complete the OC tended to decrease. It would be interesting to know how the participants ambulated with the two knee 
joint types on each section of Hafner et al.'s OC since these participants navigated over a combination of level grass, wood chips, sand, a cement ramp, and stairs [5].

In contrast to Hafner et al.'s findings, Seymour et al. reported that only the total time taken to complete the SWOC was significantly lower with the C-Leg, and this under both conditions: hands-free and carrying a $4.5 \mathrm{~kg}$ laundry basket [2]. All other parameters became nonsignificant during the loaded condition. These latter results suggest that the differences between nonmicroprocessorcontrolled knee joints and the C-Leg diminish under more demanding walking circumstances, results that seem to be supported through our study.

We observed a significant increase in THBI for the MLT condition compared with the no-MLT condition for persons using the C-Leg, suggesting that the C-Leg reduced movement efficiency when participants were subjected to an MLT. THBI is calculated by measuring heart beats per meter; hence, the THBI increase is attributable to higher heart rates only since the length of the OC did not change from one trial to the next. The reasons for this significant increase in heart rate during the MLT with the C-Leg are not known.

All participants completed the OC faster the second time when MLT was administered. The lack of randomization may have led to faster times with the MLT based on the users' prior experience with the OC. However, these times were not significantly different than the times to complete the OC without the MLT, but there were trends in this direction. There were no falls recorded during the MLT round. Falling may not have occurred with the MLT because it was always administered during the second pass, and therefore participants would anticipate and know how to accommodate those sections that caused them to fall without the MLT. It is unlikely that the faster walking speeds during the MLT trials (second round) of the OC were responsible for the increased THBI for the C-Leg. Times to complete the OC were not significantly reduced for the MLT condition with any of the knees, but only the C-Leg resulted in a significantly higher THBI (lower movement efficiency) when participants traversed the course with the MLT.

\section{STRENGTHS AND LIMITATIONS OF STUDY}

The internal validity of the study design, notably of the prosthesis itself, was controlled through the fabrication of a test prosthesis that incorporated a duplication of the participant's current socket using standard techniques (without any objective measures to confirm the duplication). The same prosthetic foot was used for all three knee types tested. Additional control of confounding variables was achieved by having the same prosthetist fabricate, fit, and align the test prostheses and adjust the knee joints. Therefore, the only difference in the prostheses between testing conditions was the prosthetic knee joint, enabling greater confidence that the differences observed in the outcome measures could be solely attributable to the knee type.

The duration of accommodation time with different prosthetic knees varies greatly between previous studies, ranging from approximately 10 hours [22] to 32 weeks [5]. English et al. reported that, after replacing the prosthetic knee joint in a single transfemoral prosthesis user, the subject's gait-related parameters stabilized after 1 week [11]. However, English et al. recommended that for research purposes a minimum of 3 weeks be used for subject accommodation [11]. Therefore, we decided to provide participants with a 4 week accommodation period, but this still may not have been sufficient for them to fully accommodate to a more complex knee joint like the C-Leg and to realize all of the potential benefits that it is claimed to afford.

The setup of the OC within a laboratory environment has both notable strengths and limitations. All of the OC sections were intentionally constructed to achieve a specific challenge and did not necessarily reflect those particular surfaces subjects were likely to encounter in everyday life. This setup allowed for an objective time assessment for all participants under similar conditions (i.e., no weather influences and an even distribution of light, temperature, and surface properties) that otherwise would not have been possible if a real-world OC setup have been used. However, the sections of the OC were relatively short, making accurate determinations of traverse times on each section more difficult. Ideally, an OC should be constructed that requires subjects to spend longer walking on each surface type, making differences in prosthetic knee performance easier to discern.

The movement efficiency measured by the THBI may also have some limitations. We deliberately designed our protocol to measure only a single trial of each condition (i.e., without MLT and with MLT) to simulate a "real-life" situation of the prosthesis user encountering different walking conditions he or she may not have previous experienced or received adequate training for. We were concerned that, if subjects were permitted multiple trials with each knee type, the results would reflect a potential order 
effect caused by their learning how to navigate each section. Additionally, we chose to use a single OC trial for each knee joint because we did not want to fatigue the participants, which potentially would have affected their performance during subsequent trials. This choice may have influenced the results since repeated measures would have made the data analysis more robust. Another limitation with the measured heart beats was that the Polar model estimated the number of heart beats by first calculating a heart rate over a $5 \mathrm{~s}$ time interval. If we had used full electrocardiography (ECG), then we could have measured the number of heart beats directly and observed how the rate changed from one OC section to the next. Nonetheless, differences in the results obtained by collecting a continuous record of ECG compared with averaging the heart rate over $5 \mathrm{~s}$ intervals presumably would be small.

The MLT test that was employed, although widely recognized and used in cognitive and behavioral studies [23-26], may have inadvertently introduced a bias affecting subject performance. In particular, this type of MLT may favor people with greater mathematical competency. However, because we used the MLT to distract participants' attention from the walking activity, the effect of this possible bias is considered minimal.

The relatively small sample size of 12 participants is a potential limitation of our investigation since it is possible that the study is underpowered. On the other hand, a larger sample size may have simply confirmed our results and indicated few, if any, statistically significant differences between variables. Future studies of this type should aspire to greater numbers of research subjects, possibly by conducting multicenter trials. However, doing so would potentially weaken the internal validity of such a study since more study personnel would be involved in similar roles. Nonetheless, feasible protocol designs must weigh the pros and cons of these types of collaborative efforts that could provide valuable information to increase our knowledge base, positively effect clinical practice, and ultimately improve quality of care for the prosthesis user.

\section{CONCLUSIONS}

Participants performed similarly on the ramp, corner, and step sections of the OC for the different test conditions. That is, no statistically significant differences were found between the different prosthetic knee joints or for the tasks undertaken. Within the foam, slalom, and rock sections, only knee joint type had an influence on the subjects' performance. During these sections, participants fitted with the C-Leg performed consistently similar to when they were fitted with the SNS. The beanbag section (i.e., simulated sand section) was the only section in which the MLT alone had a significant influence regardless of the prosthetic knee joint fitted. In other words, while the knee joint type had no effect on performance, participants were observed to walk significantly faster during the MLT. Two falls occurred with the C-Leg on the beanbag section, and one occurred with the 3R60. All falls are believed to be attributable to the relatively higher surface compliance, which appears to be an important factor that adversely affects a prosthesis user's ability to stabilize and control some prosthetic knee joints that provide stance control. A roughly 10 percent increase in THBI (i.e., decreased movement efficiency) was observed in participants wearing a C-Leg when completing the OC with an MLT. This increase in THBI for the C-Leg was statistically significant. The THBI was observed to be similar with and without MLT while participants walked with the 3R60 and the SNS. The C-Leg's reduced movement efficiency when participants walked with an MLT has clinical relevance, as many tasks in daily life are performed in parallel, such as walking and talking. Based on these results, such a task would potentially fatigue a person to a greater extent while wearing a C-Leg than while walking with either the 3R60 or the SNS knee. Additional studies are required to better understand the prosthesis user's psychological influence on perception and performance while walking on different terrains, enabling the identification of specific improvements to knee joint designs and to the development of more effective gait training protocols.

\section{ACKNOWLEDGMENTS}

\section{Author Contributions:}

Study concept and design: M. R. Meier. Acquisition of data: M. R. Meier, A. H. Hansen.

Analysis and interpretation of data: M. R. Meier, A. H. Hansen, S. A. Gard, A. K. McFadyen.

Drafting of manuscript: M. R. Meier, A. H. Hansen, S. A. Gard, A. K. McFadyen.

Critical revision of manuscript for important intellectual content: M. R. Meier, A. H. Hansen, S. A. Gard.

Final approval for manuscript to be published: M. R. Meier, A. H. Hansen, S. A. Gard, A. K. McFadyen.

Financial Disclosures: The authors have declared that no competing interests exist. 
Funding/Support: This material is based on work supported by the VA, Veterans Health Administration, Office of Research and Development, Rehabilitation Research and Development Service.

Additional Contributions: The authors express their sincere thanks to the participants for their time and dedication to this rather demanding study. Further, the authors also thank (in alphabetical order) Sara R. Koehler, MS; Pinata H. Sessoms, PhD; Steven A. Steer, MS; and Rebecca Stine, MS, VACMARL manager, for their assistance with data acquisition. In addition, the authors express their appreciation for the valuable assistance with participant recruitment received from Gregory Dimas, MD, Chief of Rehabilitation VA Lakeside, and Todd Kuiken, $\mathrm{MD}, \mathrm{PhD}$, Director of the Neural Engineering Center for Artificial Limbs, Rehabilitation Institute of Chicago. The authors would like to acknowledge the use of the VACMARL for data collection.

Institutional Review: Persons that fulfilled the eligibility criteria and expressed an interest in participating went through an informed consent process (including signing a consent form) approved by Northwestern University Institutional Review Board prior to study enrollment.

Participant Follow-Up: The authors do not plan to inform participants of the publication of this study.

Disclaimer: The views expressed in this article are those of the authors and do not necessarily reflect the position or policy of the VA or the United States government.

\section{REFERENCES}

1. Highsmith MJ, Kahle JT, Bongiorni DR, Sutton BS, Groer S, Kaufman KR. Safety, energy efficiency, and cost efficacy of the C-Leg for transfemoral amputees: A review of the literature. Prosthet Orthot Int. 2010;34(4):362-77.

[PMID:20969495]

http://dx.doi.org/10.3109/03093646.2010.520054

2. Seymour R, Engbretson B, Kott K, Ordway N, Brooks G, Crannell J, Hickernell E, Wheeler K. Comparison between the C-Leg microprocessor-controlled prosthetic knee and non-microprocessor control prosthetic knees: A preliminary study of energy expenditure, obstacle course performance, and quality of life survey. Prosthet Orthot Int. 2007;31(1): 51-61. [PMID:17365885] http://dx.doi.org/10.1080/03093640600982255

3. Kahle JT, Highsmith MJ, Hubbard SL. Comparison of nonmicroprocessor knee mechanism versus C-Leg on Prosthesis Evaluation Questionnaire, stumbles, falls, walking tests, stair descent, and knee preference. J Rehabil Res Dev. 2008;45(1):1-14. [PMID:18566922]

http://dx.doi.org/10.1682/JRRD.2007.04.0054

4. Schmalz T, Blumentritt S, Marx B. Biomechanical analysis of stair ambulation in lower limb amputees. Gait Posture. 2007;25(2):267-78. [PMID:16725325] http://dx.doi.org/10.1016/j.gaitpost.2006.04.008

5. Hafner BJ, Willingham LL, Buell NC, Allyn KJ, Smith DG. Evaluation of function, performance, and preference as transfemoral amputees transition from mechanical to microprocessor control of the prosthetic knee [Erratum appears in Arch Phys Med Rehabil. 2007;88(4):544]. Arch Phys Med Rehabil. 2007;88(2):207-17. [PMID:17270519] http://dx.doi.org/10.1016/j.apmr.2006.10.030

6. Taylor M, Gunther J. Standard walking obstacle course: Preliminary reliability and validity of a functional measurement tool. J Rehabil Outcomes Measures. 1998;2:15-25.

7. Smith DG, Willingham LL, Allyn KJ, Buell NC, Hafner BJ. Functional evaluation of the transition from a nonmicroprocessor controlled prosthesis to a microprocessor controlled prosthesis for transfemoral amputees: Early results of a clinical trial. 11th World Congress of the International Society for Prosthetics and Orthotics (ISPO); 2004 Aug 1-6; Hong Kong, China. p. 155.

8. Schmalz T, Blumentritt S, Jarasch R. Energy expenditure and biomechanical characteristics of lower limb amputee gait: The influence of prosthetic alignment and different prosthetic components. Gait Posture. 2002;16(3):255-63. [PMID:12443950] http://dx.doi.org/10.1016/S0966-6362(02)00008-5

9. Orendurff MS, Segal AD, Klute GK, McDowell ML, Pecoraro JA, Czerniecki JM. Gait efficiency using the CLeg. J Rehabil Res Dev. 2006;43(2):239-46.

PMID:16847790] http://dx.doi.org/10.1682/JRRD.2005.06.0095

10. Van de Veen PG. Above-knee prosthesis technology. Enschede (the Netherlands): P.G. Van de Veen Consultancy; 2001.

11. English RD, Hubbard WA, McElroy GK. Establishment of consistent gait after fitting of new components. J Rehabil Res Dev. 1995;32(1):32-35. [PMID:7760265]

12. Hood VL, Granat MH, Maxwell DJ, Hasler JP. A new method of using heart rate to represent energy expenditure: The Total Heart Beat Index. Arch Phys Med Rehabil. 2002;83(9):1266-73. [PMID:12235607] http://dx.doi.org/10.1053/apmr.2002.34598

13. Otto Bock 2003. Introduction to the Otto Bock computer leg. C-Leg practitioner training course. Minneaoplis (MN): 2003.

14. Thies SB, Ashton-Miller JA, Richardson JK. What causes a crossover step when walking on uneven ground? A study in healthy young women. Gait Posture. 2007;26(1):156-60. [PMID:17045479] http://dx.doi.org/10.1016/j.gaitpost.2006.08.011

15. Niang AE, McFadyen BJ. Effects of physical activity level on unobstructed and obstructed walking in young male adults. Gait Posture. 2005;22(1):75-81. [PMID:15996596] http://dx.doi.org/10.1016/j.gaitpost.2004.07.003

16. Yang BS, Ashton-Miller JA. Stepping onto raised, laterally compliant structures: A biomechanical study of age and gender effects in healthy adults. Hum Factors. 2006; 
JRRD, Volume 49, Number 4, 2012

48(2):207-18. [PMID:16884043]

http://dx.doi.org/10.1518/001872006777724480

17. Thies SB, Richardson JK, Demott T, Ashton-Miller JA. Influence of an irregular surface and low light on the step variability of patients with peripheral neuropathy during level gait. Gait Posture. 2005;22(1):40-45.

[PMID:15996590] ttp://dx.doi.org/10.1016/j.gaitpost.2004.06.006

18. MacLellan MJ, Patla AE. Adaptations of walking pattern on a compliant surface to regulate dynamic stability. Exp Brain Res. 2006;173(3):521-30. [PMID:16491406] http://dx.doi.org/10.1007/s00221-006-0399-5

19. Marigold DS, Patla AE. Age-related changes in gait for multi-surface terrain. Gait Posture. 2008;27(4):689-96. [PMID:17962018] http://dx.doi.org/10.1016/j.gaitpost.2007.09.005

20. Yang J, Jin D, Ji L, Wang R, Zhang J, Fang X, Zhou D, Wu M. The reaction strategy of lower extremity muscles when slips occur to individuals with trans-femoral amputation. J Electromyogr Kinesiol. 2007;17(2):228-40. [PMID:16603384] http://dx.doi.org/10.1016/j.jelekin.2006.01.013

21. Sjödahl C, Jarnlo GB, Persson BM. Gait improvement in unilateral transfemoral amputees by a combined psychological and physiotherapeutic treatment. J Rehabil Med. 2001; 33(3):114-18. [PMID:11482351] http://dx.doi.org/10.1080/165019701750165934

22. Johansson JL, Sherrill DM, Riley PO, Bonato P, Herr H. A clinical comparison of variable-damping and mechanically passive prosthetic knee devices. Am J Phys Med Rehabil. 2005;84(8):563-75. [PMID:16034225] http://dx.doi.org/10.1097/01.phm.0000174665.74933.0b

23. Gulich M, Zeitler HP. [The walking-counting test. A simple test for assessing the risk of falling]. Dtsch Med Wochenschr.
2000;125(9):245-48. German. [PMID:10742815]

http://dx.doi.org/10.1055/s-2007-1024084

24. Baddeley A. Working memory: Looking back and looking forward [Review]. Nat Rev Neurosci. 2003;4(10):829-39. [PMID:14523382] http://dx.doi.org/10.1038/nrn1201

25. Hausdorff JM, Schweiger A, Herman T, Yogev-Seligmann G, Giladi N. Dual-task decrements in gait: Contributing factors among healthy older adults. J Gerontol A Biol Sci Med Sci. 2008;63(12):1335-43. [PMID:19126846] http://dx.doi.org/10.1093/gerona/63.12.1335

26. Srygley JM, Mirelman A, Herman T, Giladi N, Hausdorff JM. When does walking alter thinking? Age and task associated findings. Brain Res. 2009;1253:92-99. [PMID:19084511] http://dx.doi.org/10.1016/j.brainres.2008.11.067

Submitted for publication May 18, 2010. Accepted in revised form October 5, 2011.

This article and any supplementary material should be cited as follows:

Meier MR, Hansen AH, Gard SA, McFadyen AK. Obstacle course: Users' maneuverability and movement efficiency when using Otto Bock C-Leg, Otto Bock 3R50, and CaTech SNS prosthetic knee joints. J Rehabil Res Dev. 2012;49(4):583-96.

http://dx.doi.org/10.1682/JRRD.2010.05.0094

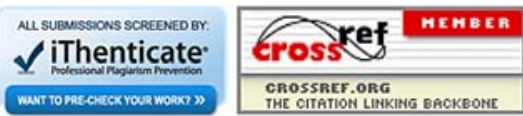

